\title{
Formação e prática de enfermeiros para a Atenção Primária à Saúde - avanços, desafios e estratégias para fortalecimento do Sistema Único de Saúde
}

\author{
Training and practice of nurses for Primary Health Care - advances, \\ challenges, and strategies to strengthen the Unified Health System
}

Elaine Thumé1, Amanda Cavada Fehn², Sonia Acioli3, Maria Elizabeth Gastal Fassa4

DOI: 10.1590/0103-11042018S118

1 Universidade Federal de Pelotas (UFPEL) - Pelotas (RS), Brasil.

Orcid: http://orcid. org/0000-0002-1169-

8884

elainethume@gmail.com

2 Universidade do Estado do Rio de Janeiro (Uerj) Rio de Janeiro (RJ), Brasil. Orcid: https://orcid. org/0000-0001-88394929

acfehn@gmail.com

3 Universidade do Estado do Rio de Janeiro (Uerj) Rio de Janeiro (RJ), Brasil. Orcid: http://orcid. org/0000-0002-07728235

soacioli@gmail.com

4 Universidade Federal de Pelotas (UFPEL) - Pelotas (RS), Brasil.

Orcid: https://orcid

org/0000-0002-2839-

3761

elizabeth.fassa@gmail.com
RESUMO Para o alcance dos Objetivos de Desenvolvimento Sustentável até 2030, o investimento em recursos humanos para a saúde é um dos componentes essenciais. Contudo, a formação desses recursos requer equilíbrio entre a oferta, a demanda e a capacidade dos profissionais para satisfazer às necessidades de atenção à saúde. A Atenção Primária à Saúde (APS) é a estratégia apoiada internacionalmente como elemento central e orientador de políticas que fortalecem o sistema de saúde. Ao celebrar 40 anos de Alma Ata e 30 anos do Sistema Único de Saúde, este artigo revisitou a história recente e destacou o profissional enfermeiro com um papel central para a consolidação da APS, sobretudo pelo potencial inovador, criativo e versátil. Os elementos aqui explorados são resultados de debates realizados pela Rede de Pesquisa em APS e reforçam a importância da construção de uma agenda política estratégica diante dos desafios presentes no cenário nacional e internacional, reafirmando a defesa da saúde e da educação pública. Organizado em três eixos, o texto aborda a expansão da enfermagem nas últimas décadas, o investimento na ampliação de escolas para formação de enfermeiros e finaliza com os desafios das práticas de enfermagem voltada à APS e potenciais estratégias de enfrentamento e melhoria, para garantir um futuro já presente no cuidado de indivíduos e populações.

PALAVRAS-CHAVE Capacitação profissional. Enfermagem de atenção primária. Atenção Primária à Saúde. Sistema Único de Saúde.

ABSTRACT To achieve the Sustainable Development Goals until 2030, investment in human resources for health is one of the essential components. However, the formation of those resources requires a balance between the supply, the demand, and the ability of professionals to meet health care needs. Primary Health Care (PHC) is the internationally supported strategy as the central and guiding element of policies that strengthen the health system. Celebrating 40 years of Alma Ata and 30 years of Brazil's Unified Health System, this article revisits the recent history and emphasizes the professional nurse with a central role for the consolidation of PHC, especially by its innovative, creative, and versatile potential. The elements explored here are results of debates carried out by the PHS Research Network and reinforce the importance of building a strategic 
political agenda facing the challenges present in the national and international scenario, reaffirming the defense of public health and education. Organized in three axes, the text addresses the expansion of nursing in the last decades, the investment in expanding schools for training nurses, and ends with the challenges of nursing practices focused on PHC and potential coping and improvement strategies, to ensure a future already present in the care of individuals and populations.

KEYWORDS Professional training. Primary care nursing. Primary Health Care. Unified Health System.

\section{Introdução}

Após quatro décadas da conferência de Alma Ata e três décadas da promulgação da Constituição Brasileira, é importante analisar a situação atual e as perspectivas na formação dos trabalhadores de saúde para atender às novas demandas decorrentes das mudanças demográficas e do perfil de morbimortalidade da população. A atenção primária é a estratégia apoiada internacionalmente como elemento central e orientador de políticas que fortalecem os Sistemas Nacionais de Saúde, com abordagem integral dos indivíduos e famílias; e compreende a saúde como resultado das suas condições de vida e de trabalho. Na agenda para o alcance dos Objetivos de Desenvolvimento Sustentável (ODS) até 2030, o investimento em recursos humanos para a saúde é um dos componentes essenciais $\mathbf{1}$.

A formação de uma Força de Trabalho em Saúde (FTS) com capacidade de responder às prioridades do século XXI requer equilíbrio entre a oferta e a capacidade dos profissionais para satisfazer às necessidades de atenção à saúde, atuais e futuras ${ }^{2}$. O descompasso entre o número de trabalhadores de saúde necessários para fornecer serviços essenciais (necessidade), a disponibilidade de profissionais de saúde (oferta) e a capacidade dos países para empregá-los (demanda) configura-se como importante obstáculo para a melhoria do desempenho e da eficiência dos serviços e sistemas de saúde s.4. $^{\mathbf{3} 4}$.

A enfermagem é uma das profissões da área da saúde com papel central para a consolidação da Atenção Primária à Saúde (APS), sobretudo pelo potencial inovador, criativo e versátil dos profissionais. O trabalho na promoção da saúde, na prevenção de doenças e na oferta de cuidados, especialmente em áreas rurais e carentes, são elementos destacados em documentos oficiais da Organização Mundial da Saúde (OMS) e pela Organização Pan-Americana da Saúde (Opas) ${ }^{5}$.

As mudanças nas diretrizes curriculares implementadas nos cursos de enfermagem têm o propósito de formar profissionais voltados a contribuir para a efetivação dos princípios e diretrizes do Sistema Único de Saúde (SUS). A profissão, com crescimento exponencial na última década, tem agregado conhecimentos, habilidades e atitudes para atuar em áreas como a atenção, a gestão, o ensino, a pesquisa e o controle social, aumentando, assim, a responsabilidade dos profissionais para com a sociedade. Entretanto, é necessário estar atento para a qualidade do ensino em um processo de expansão da participação do setor privado e crescimento sem planejamento em todo o Brasile.

$\mathrm{O}$ artigo é resultado de debates realizados pela Rede de Pesquisa em APS e reforça a 
importância da construção de uma agenda política estratégica para a APS no SUS ante os desafios ora presentes no cenário nacional e internacional, reafirmando a defesa da saúde e da educação. Organizado em três eixos, o texto aborda a expansão da enfermagem nas últimas décadas, o investimento na ampliação de escolas para formação de enfermeiros e finaliza com os desafios das práticas de enfermagem voltada à APS, de modo a garantir um futuro já presente no cuidado de indivíduos e populações.

\section{A expansão da enfermagem no contexto do movimento da Reforma Sanitária e da Estratégia Saúde da Família no processo de formação dos enfermeiros}

No Brasil, no início dos anos 1970, o número de enfermeiros e o número de vagas eram insuficientes para atender ao proposto no Plano Decenal de Saúde para as Américas. A concentração dos cursos na região Sudeste e em instituições privadas dificultava o acesso às instituições de ensino. Para o enfrentamento dessa realidade, foi determinado o aumento no número de vagas para enfermagem na rede superior pública, sendo criadas escolas de enfermagem vinculadas a Universidades Federais em todas as regiões do País. Em paralelo, ocorreu o investimento na qualificação dos docentes em cursos de pós-graduação no nível de mestrado e doutorado, demandando a representação da área na Coordenação de Aperfeiçoamento de Pessoal de Nível Superior (Capes) ${ }^{7}$.

No âmbito mundial, o movimento que culminou com a declaração de Alma Ata, defendia a necessidade de ampliar a oferta e investir nos cuidados primários à saúde como alternativa ao modelo de saúde vigente. Os padrões de morbimortalidade poderiam ser enfrentados com o forte investimento em ações de prevenção às doenças e promoção da saúde com opções políticas de largo alcance populacional com articulação intersetorial, de modo a diminuir as desigualdades econômicas e sociais ${ }^{5}$.

O movimento da Reforma Sanitária, no Brasil, conseguiu garantir o direito à saúde como sendo fundamental dos indivíduos e populações na Constituição Federal de 1988, com a criação do SUS. Entre as prerrogativas para garantir o acesso universal, público e gratuito, atribuiu-se ao SUS a ordenação da formação de recursos humanos na área da saúde e o desenvolvimento científico e tecnológico, tema recorrente e reforçado nas Conferências Nacionais de Saúde subsequentes ${ }^{8}$.

Em 2001, a publicação das Diretrizes Curriculares Nacionais do Curso de Graduação em Enfermagem (DCN/ENF) (9) estimulou reformas nas Instituições de Ensino Superior (IES) em todo o Brasil; e dentre as competências e habilidades gerais requeridas para o exercício profissional, destaca-se o aprendizado que valoriza questões ético-humanistas quanto à atenção à saúde, tomada de decisões, capacidade de comunicação e liderança. As competências de ordem técnica estão relacionadas com a administração e gerenciamento e educação permanente dos profissionais ${ }^{\mathbf{1 0}}$.

Em 2003, a Política Nacional de Formação e Desenvolvimento para o SUS" aprovou investimentos na educação permanente em saúde com estímulo para o engajamento das instituições de ensino na área da saúde. No ano seguinte, foi instituída a Política Nacional de Educação Permanente em Saúde, com incentivo a criação de Polos regionais integrando gestores, trabalhadores, instituições de ensino, negociações interinstitucionais e intersetoriais ${ }^{\mathbf{1 2}}$.

Na perspectiva de consolidação do SUS, essas iniciativas foram importantes em um contexto de investimentos na ampliação do modelo de atenção com base na saúde da família, principalmente em municípios de grande porte, ocorrido com o Programa 
de Consolidação e Ampliação da Saúde da Família (Proesf). O ingresso de enfermeiros assistenciais nas equipes de saúde foi um marcador essencial de mudança no modelo de atenção, tanto para a proposta de ampliação do Programa Saúde da Família (PSF) quanto dos Agentes Comunitários de Saúde (ACS). Os municípios passaram a ser um importante empregador e os processos de municipalização da saúde ampliados ${ }^{13}$.

A expansão do Programa de Agentes Comunitários (Pacs) e do PSF, com início, respectivamente, em 1991 e 1994, além de uma alternativa para a mudança de paradigma no modelo de atenção, constituiu-se como um crescente mercado de trabalho para profissionais médicos e de enfermagem. Em setembro de 2017, dados fornecidos pelo Ministério da Saúde registravam a existência de 41.619 Equipes de Saúde da Família (EqSF) em atividade no Brasil com cobertura de $63 \%$ da população, com o apoio de 263.236 ACS, além de 4.593 Núcleos de Apoio à Saúde da Família (Nasf). Destaque também para $89 \mathrm{EqSF}$ atendendo populações ribeirinhas e seis Unidades Básicas de Saúde (UBS) fluviais. Esses indicadores de capacidade instalada apontam para a crescente importância da atenção primária no Brasil, com a expansão na cobertura de médicos, enfermeiros e outros profissionais de saúde no território nacional, constituindo-se em um expoente mercado de trabalho ${ }^{\mathbf{1 4}}$.

Essa expansão no número de equipes cadastradas ocorreu também como consequência da implementação do Programa Mais Médicos (PMM), em 2013, que inseriu na agenda política ações voltadas à superação da má distribuição e escassez de profissionais médicos no território nacional e abriu precedente para a ampliação da rede de cuidados em APS. O programa ampliou a oferta de profissionais médicos em cerca de $18 \mathrm{mil}$ novos trabalhadores, sendo a maioria recrutada mediante acordo de cooperação internacional entre a Opas, o governo de Cuba e o governo brasileiro ${ }^{15}$, com importantes reflexos para a ampliação da contratação de enfermeiros, técnicos de enfermagem e ACS. Além do recrutamento, a política também incluiu a expansão das escolas de medicina no País e a formação especializada em saúde da família.

Por outro lado, a Política Nacional de Atenção Básica (PNAB), publicada em setembro de 2017, revogou a prioridade do modelo ESF, embora o reafirme como estratégia prioritária para expansão e consolidação da atenção básica ${ }^{16}$. Ao modificar o financiamento das equipes incluindo a flexibilização da carga horária dos profissionais e a opcionalidade da presença e do número de ACS nas equipes, descaracteriza o modelo de atenção defendido nos últimos 25 anos $^{16}$.

\section{A evolução da formação de enfermeiros no Brasil}

Dados da Relação Anual de Informações Sociais (Rais), ao analisar os empregos formais de profissões e ocupações de saúde, identificam 2.204.173 empregos no setor, sendo 1.901.423 nas atividades componentes do macrossetor (núcleo, complementares, indústria, comércio, financiamento e saneamento) e 302.750 nos demais setores. Aquelas que representam os maiores contingentes de emprego foram os auxiliares de enfermagem (355.824), médicos (240.428), técnicos em enfermagem (239.904), ACS (237.548) e enfermeiros (129.905) 17.

No Brasil, a primeira década dos anos 2000 demarca um aumento da proporção de todos os profissionais de saúde por número de habitantes (hab.). No âmbito da enfermagem, em 2000, havia 4,4 enfermeiros/10 mil hab. Dez anos depois, essa taxa era de 15,1/10 mil hab. A mudança na oferta de técnicos e auxiliares de enfermagem também foi observada, passando de 22,9 técnicos ou auxiliares de enfermagem/10 mil hab. em 2000, para 60,8 profissionais/mil hab. O Projeto de Profissionalização dos Trabalhadores da 
Área da Enfermagem (Profae), que ocorreu entre 2000 e 2007, é apontado como responsável pelo incremento ${ }^{18}$.

A expressividade numérica da profissão da enfermagem impressiona ao corresponder por praticamente metade do total de trabalhadores da área da saúde. Os enfermeiros representam $24 \%$ dos profissionais com registro no Conselho Federal de Enfermagem (Cofen) ${ }^{19}$. Estão presentes em todos os municípios do Brasil; e cerca de $60 \%$ tem vínculo empregatício com o setor público de saúde, com inserção nas UBS ou em cargos de gestão em secretarias municipais e estaduais de saúde 20 .
Dados do Cofen possibilitam verificar a expressividade numérica da profissão da enfermagem no Brasil, com 2.066.302 inscrições ativas em junho 2018, das quais: 498.110 enfermeiros, 1.148.840 técnicos, 419.008 auxiliares e 344 obstetrizes $^{19}$.

No período de 2001 a 2011, foi observado um incremento no número de escolas de enfermagem tanto em instituições privadas quanto públicas. $\mathrm{O}$ aumento do número de vagas, as quais impactam pela dimensão, superam o total de $192 \mathrm{mil}$ assentos em escolas de enfermagem públicas e privadas ofertados no período de 2002 a 2014 (gráfico 1).

Gráfico 1. Evolução do total de vagas ofertadas nos cursos públicos e privados de enfermagem no período de 2000 a 2014. Brasil, 2017

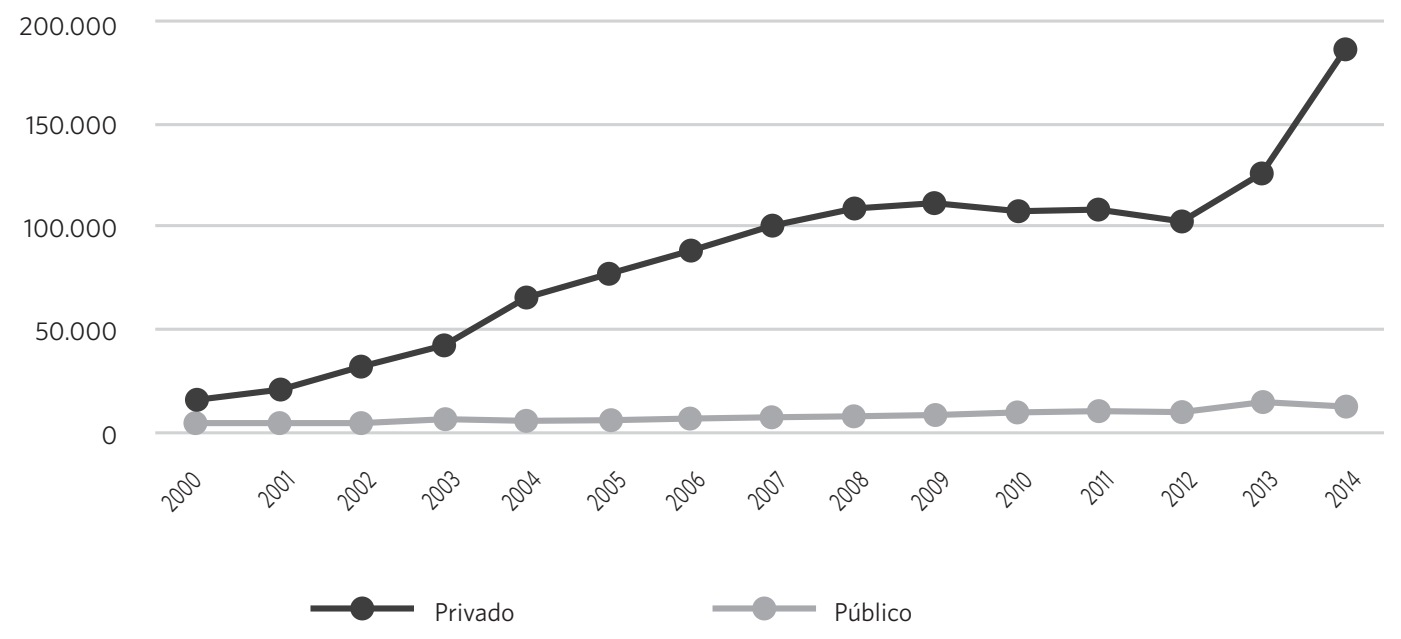

Fonte: Sigras-2017

No período de 2001 a 2011, foi observado um incremento de 393\% do número de escolas de enfermagem nas instituições privadas em comparação ao incremento de $122 \%$ em relação ao número de escolas públicas. Em 2011, havia 826 cursos de graduação em enfermagem, 160 em IES públicas e 666 em IES privadas, ou seja, o número de vagas em estabelecimentos privados ultrapassa em mais de cinco vezes as do setor público ${ }^{\mathbf{1 7}, 21}$. Em 2012, esse número foi de 838 cursos; e, em 2013, atingiu $888^{22}$, com mais de 180 mil vagas (presenciais e à distância)em escolas privadas de enfermagem em 2014. 
Esta tendência observada na enfermagem acompanha as características do sistema de ensino superior no Brasil, em que há o predomínio de instituições privadas $(70 \%$ a $80 \%)^{17}$. A região Sudeste apresenta maior número de cursos de graduação em enfermagem seguida das regiões Nordeste, Sul, Centro-Oeste e, por fim, da região Norte. O cálculo da taxa de crescimento aponta que, no período de 2001 a 2011, as regiões Centro-Oeste e Nordeste apresentaram o maior percentual de ascensão no número de cursos, seguido das regiões Sudeste, Norte e Sul. Entretanto, com relação à taxa de crescimento do número de vagas, a região que mais cresceu foi a Centro-Oeste, seguida das regiões Norte e Nordeste. Os estados de São Paulo, Minas Gerais, Espírito Santo e Rio de Janeiro detêm mais de $54 \%$ do total de vagas dos cursos de graduação em enfermagem ${ }^{17}$.

Em 2000, o número de concluintes era maior nas regiões Sudeste e Nordeste e menor no Centro-Oeste e Norte. Passados dez anos, não ocorreu alteração nesse quadro, com exceção da região CentroOeste, que ultrapassou a região Norte em número de concluintes. No período de 2000 a 2011, o número de vagas era ainda superior ao de egressos. E assim permanece atualmente. No Brasil, o número de concluintes não acompanha o número de vagas, o que permite supor que elas não são preenchidas por completo e/ou há um alto percentual de desistência durante o curso ${ }^{17}$.

Na pós-graduação, também foi observado o crescimento no período de 2006 a 2016, principalmente dos programas de doutorado e de mestrado profissional. Em 2016, havia um total de 76 programas de pós-graduação na área da enfermagem (36 de mestrado e doutorado, 2 de doutorado, 15 de mestrado acadêmico e 23 de mestrado profissional), representando um aumento de $17 \%$ no número de programas de pós-graduação entre 2013 e 2016 com expansão para as regiões Sul e Nordeste, persistindo a carência nas regiões Norte e Centro-Oeste ${ }^{23}$.
Um dos elementos que destacam a importância do setor público é a pós-graduação; responsável por $93 \%$ da titulação de doutores, $87 \%$ de mestres acadêmicos, $59 \%$ de mestres profissionais e $77 \%$ em programas de residência ${ }^{24}$. O aumento gradual na titulação de mestres e doutores ocorreu a partir da segunda metade dos anos 1990. Ressalta-se ainda o período de 1975 a 2016, com titulação de 11.285 mestres acadêmicos, 3.358 doutores e 786 mestres profissionais ${ }^{23}$.

Quanto às vagas em IES de Enfermagem, cabe destacar a existência da modalidade de Ensino a Distância (EaD). Dois momentos estimularam a abertura de cursos de enfermagem a distância: i) A Lei de Diretrizes e Bases da Educação (LDB) de $1996^{25}$, pela autonomia e fomento a cursos utilizando esta metodologia para a graduação, cursos de extensão, sequenciais, pós-graduação, educação de jovens e adultos, aperfeiçoamento de professores e educação continuada; ii) a partir de 1998, com o cadastramento pelo Ministério da Educação e Cultura (MEC) de IES interessadas em oferecer cursos à distância ${ }^{26}$.

O aumento de cursos de graduação em enfermagem, presenciais e a distância, observado no Brasil tem sido considerado excessivo, pois, ainda que o crescimento no número de vagas e cursos de graduação em enfermagem indique uma expansão da profissão, coloca-se permanentemente a preocupação com a qualificação e a regulação desse processo. Os Conselhos Regionais de Enfermagem e entidades representativas da categoria, como a Associação Brasileira de Enfermagem (Aben), vêm se manifestando de maneira contrária à formação de enfermeiros com atividades presenciais limitadas.

Além da expressividade no numerário de trabalhadores, o potencial criativo e inovador da enfermagem tem ganhado imenso destaque na agenda internacional com o movimento Nursing Now, com base no relatório do Triplo Impacto. O documento sugere contribuições singulares da enfermagem na melhoria da condição de saúde das populações, 
tendo como meta para 2020 o estabelecimento de liderança política da enfermagem e o incentivo à formação de redes de pesquisa com abrangência mundial27,28. A estratégia visa garantir que a enfermagem tenha participação nas instâncias de gestão dos sistemas de saúde na maioria dos países e o reconhecimento do papel dos enfermeiros para o alcance dos objetivos da saúde pública ${ }^{28}$.

O movimento Nursing Now retoma orientações acerca da formação dos profissionais de saúde, nomeadamente a enfermagem, já presentes em outros documentos, como 'Transforming and Scaling up health professionals education and training'29 $\mathrm{e}$ 'Global Strategic directions for strengthening nursing and midwifery' 2016-20230, ambos da OMS. Paralelamente, ações das Redes Internacionais de Enfermagem das Américas, um projeto colaborativo da Opas/ OMS por meio da Unidade de Recursos Humanos para a Saúde do Departamento de Sistemas e Serviços de Saúde, buscam impulsionar a categoria dos profissionais de enfermagem e fortalecer seu desempenho ${ }^{31}$.

No País, instituições representativas da enfermagem como a Aben e os Cofen lançam esforços também direcionados a impulsionar o protagonismo da profissão. Regionalmente, os estados do Rio de Janeiro e de Santa Catarina, por exemplo, são modelos de experiências positivas. Ambas as capitais desenvolveram protocolos de enfermagem, debatidos nos cenários político, acadêmico e social, os quais respaldam os trabalhadores legalmente e viabilizam uma conduta ampla e baseada em evidências ${ }^{32}$.

\section{Desafios e estratégias na formação e nas práticas da enfermagem na APS}

O modelo de atenção com foco na APS se distingue pela visão do indivíduo em seu contexto socioeconômico-cultural e direciona a demanda para um novo perfil de profissionais que saibam atuar diante das necessidades de saúde desse sujeito, da sua família e da comunidade em uma equipe multiprofissional e com um olhar interdisciplinar e intersetorial ${ }^{33}$.

A avaliação da estrutura atual e as estimativas futuras para a profissão da enfermagem até 2030, simula diferentes cenários ${ }^{34}$ No cenário tendencial, tendo como base o comportamento das escolas de enfermagem do País em relação ao número anual de vagas, os autores afirmam que o expressivo crescimento de $14,9 \%$, ocorrido entre os anos de 1991 e 2012, não será sustentado. Entre os anos de 2010 e 2012, as vagas decresceram a 3,38\% ao ano. Assim, supõe-se a desaceleração do crescimento até 2030 . Tendo como base um cenário regulatório, no qual haveria uma reversão do quadro atual de fechamento de vagas de modo a ampliar o escopo de práticas dos enfermeiros e sua expansão nas atividades da ESF, e considerando que mesmo que os mais de $50 \%$ da força de trabalho graduada em enfermagem (hoje sem atuar na área) voltasse a exercer a profissão, ainda assim o número de enfermeiros não seria suficiente para atender ao novo cenário regulatório suposto, com previsão de crescimento anual de $2 \%$ no número de vagas, até 2020 , e posterior congelamento das mesmas até 2025. Por fim, um terceiro cenário no qual o número de vagas anuais esperado entre 2010-2015 se manteria constante até 2025. Considerando esses cenários, a prospecção do resultado para 2030 será um estoque de enfermeiros oscilando entre 1,499 e 2,037 milhões de profissionais para Brasil.

Outra questão peculiar da enfermagem está relacionada com o perfil dos profissionais com altas proporções de mulheres e com saída do mercado de trabalho no início da terceira idade. Apesar de ter sido observado o rejuvenescimento na força de trabalho da enfermagem, com alargamento da base da pirâmide, não houve alteração no topo, diferentemente do ocorrido com padrão dos 
médicos e dentistas. Essa tendência sinaliza que a demanda por enfermeiros continuará condicionada à capacidade de acomodação do mercado, a qual se mostrou insuficiente no período anterior às estimativas, afinal mais da metade dos graduados da área não estava ocupada na própria profissão. O censo demográfico de 2010 identificou 355.383 enfermeiros, apenas $46,7 \%$ se ocupavam da profissão, apesar de 308.429 (86,8\%) estarem economicamente ativos ${ }^{34}$.

Recentemente, no XVI Seminário Nacional de Diretrizes para a Educação em Enfermagem e no XIII Simpósio Nacional de Diagnóstico de Enfermagem, promovido pela Aben no período de 05 a 08 de junho de 2018 em Florianópolis (SC), foi redigida a carta de Florianópolis que elaborou recomendações, reforçou a importância da enfermagem na defesa do SUS e os princípios democráticos e participativos na educação, mobilizando os atores sociais envolvidos nas redes de ensino-serviço que integram a formação em enfermagem ${ }^{35}$.

Dentre as recomendações da carta, há destaque para o redimensionamento da oferta de vagas nos Cursos, Faculdades e Escolas de Enfermagem, tendo as necessidades de saúde da população, do mercado de trabalho e da oferta de espaços para estudos teórico-práticos como limitadores à expansão. Foi reafirmada a importância da integração ensino-serviço-comunidade desde o início do curso nos cenários de práticas de saúde e com articulação dos trabalhadores, considerando a formação técnica, graduação e pós-graduação. Sobre o $\mathrm{EaD}$, a plenária reafirmou posição contrária a essa modalidade de ensino na área da saúde, em todos os níveis de formação (técnico, graduação e pós-graduação) ${ }^{35}$. Contudo, há necessidade de debates que considerem algumas especificidades e vantagens, nomeadamente a cursos de mestrado a distância por exemplo. O Prof Saúde, iniciativa do Ministério da Saúde com a Fundação Oswaldo Cruz e com diversas outras instituições do País, tem-se destacado como uma alternativa positiva na qualificação de profissionais e gestores do SUS na área médica.

Considerando a proposta da OMS do Movimento Nursing Now, é necessário elaborar uma matriz de habilidades e competências para enfermagem na APS, incluindo o debate sobre as práticas avançadas. As competências centrais da prática em enfermagem incluem aquelas no âmbito da prática clínica, liderança, experiência na atenção ao paciente e ao seu grupo familiar e comunitário, investimento na promoção da saúde, com colaboração entre os profissionais e decisões baseadas na ética. Outra habilidade com potencial de utilização pela enfermagem será manter e ampliar a participação e representação política. Além disso, a incorporação de novas tecnologias às práticas exige qualificação continua, aumenta a demanda efetiva da força de trabalho e interliga diferentes serviços do setor saúde ${ }^{17}$.

$\mathrm{Na}$ área da regulação, legislação e acreditação, recomenda-se o desenvolvimento de políticas de regulação das IES com padrões de qualidade em diferentes áreas, como, por exemplo, corpo docente, processo de admissão/ingresso de alunos, currículo baseado em competências, entre outros, para as instituições públicas e privadas de ensino superior. É necessário também desenvolver políticas de acreditação e legislação comprometidas com a excelência do ensino superior em saúde e orientadas para o fortalecimento do SUS e do acesso universal da saúde.

Sobre a qualidade e disponibilidade da informação sobre Recursos Humanos em Saúde (RHS), é preciso investir e incentivar o uso de Sistemas de Informação e Plataformas com dados sobre a FTS, como o Observatório de RHS, permitindo, assim, acesso e tomada de decisões baseadas em dados concretos, atuais e condizentes com a realidade. Da mesma forma, o investimento em tecnologias e-Health, como, por exemplo, o Telessaúde Enfermagem e a adequação dos prontuários eletrônicos à prática 
clínica e assistencial desses profissionais, torna-se cada vez mais imprescindível ${ }^{36,37}$. A adaptação das plataformas de registros clínicos ao processo de trabalho do enfermeiro permite o uso potencial da Sistematização da Assistência da Enfermagem (SAE). Paralelamente, garante ferramentas estratégicas de acompanhamento longitudinal das populações e melhoria contínua da qualidade, por meio de indicadores de processo e resultado, os quais podem orientar projetos de educação permanente e continuada ajustadas às necessidades locais.

No âmbito da pesquisa, da produção de conhecimento e inovação em RHS, há necessidade de realizar pesquisas aplicadas às necessidades nacionais, dialogando com as estratégias e direcionamentos internacionais na área de RHS. Dessa forma, será possível desenvolver uma agenda com base em evidências para o planejamento de ações e elaboração de políticas alinhadas aos SUS. A manutenção do financiamento de estratégias como o estágio de Vivências e Estágios na Realidade do Sistema Único de Saúde (VER-SUS) e o Programa de Educação para o Trabalho em Saúde (PET Saúde), voltados para a formação com ênfase na APS e para o incentivo dos programas de especialização nos moldes da residência, denota potencial para qualificar a FTS.

O estímulo ao empoderamento e o papel de liderança dos profissionais de enfermagem são destacados em documentos técnicos, compreendendo a profissão como elemento central para melhoria das condições de saúde das populações, promoção da equidade de gênero e apoio e fortalecimento do crescimento econômico, como sugere o Relatório 'Triple Impact'27. Os conteúdos abordados no Exame Nacional de Desempenho de Estudantes (Enade) reforçam a amplitude das competências e habilidades esperadas do profissional enfermeiro, os quais devem ser considerados no conjunto de elementos a serem avaliados, quais sejam: a infraestrutura física e de recursos humanos das IES, além de projetos pedagógicos condizentes com as diretrizes curriculares ${ }^{\mathbf{3 8}}$.

Além dos atendimentos aos indivíduos e suas famílias, ao enfermeiro também compete a aproximação com a comunidade, incluindo as sociedades e associações profissionais no debate sobre necessidades formativas. Nesse sentido, é necessário trabalhar colaborativamente com os vários atores sociais envolvidos no processo de educação e cuidado de saúde, além dos trabalhadores de saúde, os governos e as associações profissionais, independentemente da conformação do sistema nacional de saúde ${ }^{39}$.

Com relação ao mercado de trabalho em saúde, é importante alinhar as políticas de RHS às necessidades e demandas da população e do SUS, levando em consideração aspectos da dinâmica do mercado de trabalho e as novas políticas educacionais ${ }^{40}$.

A oferta de condições dignas de trabalho e satisfação profissional são desafios constantes, de modo a promover condições e contratos de trabalho compatíveis com a ética e o exercício profissional, garantindo equidade de gênero, etnia e cor. O desenvolvimento de mecanismos de auscultação dos profissionais da saúde, a fim de compreender seus níveis de satisfação e estabelecer estratégias que possam melhorar desempenho e motivação, são fundamentais neste processo².

$\mathrm{O}$ investimento massivo na qualidade da formação dos docentes nas IES públicas e privadas e no desenvolvimento de competências estratégicas e adequadas às mudanças pedagógicas propostas pelas DCN/ENF e alinhadas às necessidades da comunidade são pontos a destacar. Nas IES públicas e privadas com cursos na área da saúde, é importante garantir a existência de Departamentos de APS, Saúde Pública, Saúde Coletiva e ampliar a presença de profissionais no corpo docente com formação específica na área.

$\mathrm{Na}$ implementação das DCN/ENF, é imprescindível realizar atividades de monitoramento e avaliação das estratégias de implantação dos projetos pedagógicos, de 
modo a avaliar a efetividade delas e refletir sobre necessidade de reformulação dos processos de formação. Recomenda-se a inclusão na pauta das IES da formação multiprofissional durante a realização dos cursos de graduação e pós-graduação (modalidade residência em serviço). O fortalecimento de programas de especialização, nos moldes da residência, com projetos pedagógicos em consonância com as competências e habilidades requeridas para profissionais da APS e a inclusão dos enfermeiros nos atuais cursos de formação de preceptores ofertados pelo Ministério da Saúde também é recomendável.

\section{Considerações finais}

O suporte filosófico oferecido pela APS aos sistemas de saúde para organizarem seus serviços é flexível e se ajusta à mudança acelerada da natureza dos problemas de saúde. Eventos relacionados com o processo intenso de urbanização e globalização, com o envelhecimento da população, com o aumento da carga de doenças, com a incorporação de novas tecnologias e com o potencial de transmissibilidade, dado o dinamismo da mobilidade das sociedades atuais, entre outros fatores, torna ainda mais complexos os cuidados dos indivíduos e populações.

Essa realidade, cada vez mais frequente, impõe desafios tanto para a prestação de serviços como para a gestão do cuidado em saúde, especificamente de enfermagem, que deve ser cada vez mais integrado e abrangente. Nesse sentido, é fundamental o fortalecimento de Sistemas Nacionais de Saúde baseados na APS, os quais depende de radicais e importantes mudanças na formação dos profissionais de saúde.

Edição especial do The Lancet, em 2010, sobre a educação profissional em saúde para o século XXI ${ }^{41}$ ressalta que a instituição de saúde não pode ser considerada de forma isolada e individual, mas, sim, como parte de um conjunto inter-relacionado de organizações que implementam as diversas funções de um sistema educacional. Essa observação reforça a configuração da unidade de saúde como integrante do processo formativo ${ }^{\mathbf{4 1}}$. A implementação das mudanças curriculares pode fortalecer o desenvolvimento de boas práticas profissionais, se alcançadas, no direcionamento a uma formação profissional mais abrangente e com foco nos cuidados primários.

A formação dos profissionais de saúde deve ir além de uma simples produção e provimento de funcionários para servir um determinado sistema de saúde. Ela deve refletir, individual e coletivamente, os diferentes, mas complementares, papéis desempenhados por esses atores, os verdadeiros agentes de mudanças ${ }^{42}$. Esse novo cenário de ambientação para a formação profissional instiga a necessidade da aprendizagem como núcleo de uma educação transformadora, moldada pela adaptação de competências, as quais devem ser planejadas para contextos específicos/locais considerando o poder dos fluxos globais de informação e conhecimento ${ }^{41}$.

É evidente que essas transformações exigem uma série de outras mudanças e adaptações em diferentes níveis e setores e que há sempre resistência nos processos em que as regras são redefinidas. A inovação está em direcionar as mudanças organizacionais a partir dos profissionais de saúde e das necessidades dos sistemas de saúde, de educação e da população de forma interdependente ${ }^{41}$.

Serviços de cuidados primários proporcionam um melhor alinhamento entre as necessidades da população e custo-efetividade. Esta abordagem explora a contribuição das diferentes categorias profissionais de saúde potencializando as competências e habilidades dos trabalhadores no seu escopo de atuação evitando custos excessivos e abordagens invasivas de cuidados terciários ${ }^{3}$.

O que se observa no cenário internacional é um reforço às políticas e ações de saúde orientadas aos cuidados primários perceptível no discurso adotado na grande maioria das metas traçadas no terceiro ODS, por exemplo, 
ao enfatizar a abordagem da prevenção e promoção em saúde por meio de indicadores com evidências de impacto positivo na APS.

Por fim, consideramos que as universidades têm um papel decisivo na garantia da qualidade do cuidado por meio da formação profissional. A prática da enfermagem na APS no Brasil depende de políticas de fortalecimento da saúde como direito e da regulamentação profissional, essencial para as demandas corporativas dos diferentes profissionais da saúde e para o trabalho em equipe.

\section{Referências}

1. Washington DC. Ampliação do papel dos enfermeiros na atenção primária à saúde [internet]. Washington, DC: OPAS; 2018 [acesso em 2018 jul 9]. Disponível em: http://www.paho.org.

2. World Health Organization. Global strategy on human resources for health: Workforce 2030 [Internet]. Geneva: World Health Organization; 2016 [acesso em 2018 jul 9]. Disponível em: http://apps.who.int/iris/ bitstream/handle/10665/250368/9789241511131-eng.pdf;jsessionid=039D82637D28A282A115BA284 02C1E61?sequence=1.

3. Organização Mundial De Saúde (OMS). Relatório mundial de saúde, 2006: trabalhando juntos pela saúde. Brasília, DF: Ministério da Saúde; 2007.

4. Bacon TJ, Newton WP. Innovations in the education of health professionals. N C Med J [internet]. 2014 [acesso em 2018 jun 29]; 75(1):22-7. Disponível em: http://www.ncbi.nlm.nih.gov/pubmed/24487754.

\section{Colaboradores}

As autoras contribuíram substancialmente para a concepção e o planejamento ou para a análise e a interpretação dos dados; contribuíram significativamente na elaboração do rascunho ou revisão crítica do conteúdo; e participaram da aprovação da versão final do manuscrito.
5. Conferência Internacional sobre cuidados Primários de Saúde. Declaração de -Ata. 1978 set [acesso 2018 jun 21]. Disponível em: http://cmdss2011.org/ site/wp-content/uploads/2011/07/Declaração-Alma-Ata.pdf.

6. Dal Poz MR, Varella TC, Santos MR. Formação em saúde: problemas e tendências. In: Saúde amanhã: textos para discussão. Rio de Janeiro: Fundação Oswaldo Cruz; 2015. p. 10.

7. Paim L. A formacão de enfermeiros no Brasil na década de 70. Rev. Bras. Enferm. [internet]. 2001 [acesso em 2018 jul 9]; 53(4):186-96. Disponível em: http://www.scielo.br/pdf/reben/v54n2/v54n2a04. pdf.

8. Ceccim RB, Armani TB, Rocha CF. O que dizem a legislação e o controle social em saúde sobre a formação de recursos humanos e o papel dos gestores públicos, no Brasil. Cienc Saúde Colet. [internet]. 
2002 [acesso em 2018 jul 9]; 7(2):373-83. Disponível em: http://www.scielo.br/scielo.php?script=sci arttext\&pid=S1413-81232002000200016\&lng=pt\&t lng=pt.

9. Ribeiro JP, Tavares M, Esperidião E, et al. Análise das diretrizes curriculares: uma visão humanista na formação do enfermeiro. Rev. enferm. UERJ. [Internet]. 2005 [acesso 2018 jul 14]; 13:403-409. Disponível em: http://www.facenf.uerj.br/v13n3/v13n3al7. pdf.

10. Brasil. Conselho nacional de saúde. Resolução $n^{0}$ 335 , de 27 de novembro de 2003. Afirmar a aprovação da "Política Nacional de Formação e Desenvolvimento para o SUS: Caminhos para a Educação Permanente em Saúde" e a estratégia de "Pólos ou Rodas de Educação Permanente em Saúde," como instâncias ocorregionais e interinstitucionais de gestão da Educação Permanente. Diário Oficial da União. 5 Fev 2004.

11. Brasil. Ministério da Saúde. Portaria no 198/GM/ MS, de 13 de fevereiro de 2004. Institui a Política Nacional de Educação Permanente em Saúde como estratégia do Sistema Único de Saúde para a formação e o desenvolvimento de trabalhadores para o setor e dá outras providências. [internet]. 2004 [acesso em 2018 out 8]. Disponível em: http://bvsms. saude.gov.br/bvs/saudelegis/gm/2017/MatrizesConsolidacao/comum/13150.html.

12. Gil CRR. Formação de recursos humanos em saúde da família: paradoxos e perspectivas. Cad Saúde Pública [internet]. 2005 [acesso em 2018 jul 9]; 21(2):490-498. Disponível em: http://www.scielo. br/scielo.php?script=sci_arttext\&pid=S0102-311X2 $005000200015 \& \operatorname{lng}=\mathrm{pt} \& \operatorname{lng}=\mathrm{pt}$.

13. Dal Poz M. A crise da força de trabalho em saúde. Cad. Saúde Pública [internet]. 2013 [acesso em 2018 jul 9]; 29(10):1924-1926. Disponível em: http:// dx.doi.org/10.1590/0102-311XPE011013.

14. Campos GWS, Pereira Júnior N. Primary care in Brazil, and the Mais Médicos (More Doctors) Program in the Unified Health System: achievements and limits. Ciênc. Saúde Colet. [internet]. 2016 [acesso em 2018 jul 9]; 21(9):2655-2663. Disponível em: http://www.scielo.br/scielo.php?script=sci abstract\&pid=S1413-81232016000902655\&lng=en\& $\mathrm{nrm}=$ iso\&tlng=pt.

15. Brasil. Ministério da Saúde. Portaria $n^{0} 2.436$, de 21 de setembro de 2017. Aprova a Política Nacional de Atenção Básica, estabelecendo a revisão de diretrizes para a organização da Atenção Básica, no âmbito do Sistema Único de Saúde (SUS) [internet]. Diário Oficial da União. 22 Set 2017. [acesso em 2018 out 8] Disponível em: http://bvsms.saude.gov.br/ bvs/saudelegis/gm/2017/prt2436_22_09_2017. html.

16. Conselho Federal de Enfermagem. Enfermagem em Números [internet]. [acesso em 2018 jul 9]. Disponível em: http://www.cofen.gov.br/enfermagem-em-numeros.

17. Machado MH, Oliveira ED, Lemos W, et al. Mercado de trabalho da enfermagem: aspectos gerais. Enferm em Foco [internet]. 2016 [acesso em 2018 jul 15]; 7(Esp):35-53. Disponível: http://revista.cofen.gov.br/index.php/enfermagem/article/ view/691/301.

18. Departamento Intersindical de Estatística e Estudos Socioeconômicos - DIEESE. Aunário da Saúde do Trabalhador [internet]. 2016 [acesso em 2018 jul 16]. Disponível em: https://www.dieese.org.br/anuario/2016/Anuario_Saude_Trabalhador.pdf

19. Poz MRD, Perantoni CR, Girardi S. Formação, mercado de trabalho e regulação da força de trabalho em saúde no Brasil. In: A saúde no Brasil em 2030 - prospecção estratégica do sistema de saúde brasileiro: organização e gestão do sistema de saúde. Rio de Janeiro: Fiocruz; 2013. p. 187-233.

20. Bem vindo(a) ao SIGRAS [internet]. 2018 [acesso em 2018 out 9]. Disponível em: http://www.neonet. com.br/sigras_new_mapa/home.php.

21. Teixeira E, Fernandes JD, Andrade AC, et al. Panorama dos cursos de Graduação em Enferma- 
gem no Brasil na década das Diretrizes Curriculares Nacionais. Rev. bras. Enferm.[internet] 2013 [acesso em 2018 jul 13]; 66(esp):102-10. Disponível em: http://www.scielo.br/scielo.php?script=sci arttext\&pid=S0034-71672013000700014\&lng=pt\&t lng=pt.

22. Brasil. Ministério da Educação. Coordenação de Aperfeiçoamento de Pessoal de Nivel Superior (CAPES). Resultado da Avaliação Quadrienal 2017 avaliação quadrienal [internet]. 2017 [acesso em 2018 jul 16]. Disponível em: http://avaliacaoquadrienal.capes.gov.br/resultado-da-avaliacao-quadrienal-2017-2.

23. Machado MH, Wermelinger M, Vieira M, et al. Aspectos gerais da formação da enfermagem: o perfil da formação dos enfermeiros, técnicos e auxiliares. Enferm. foco [internet]. 2016 [acesso em 2018 jul 8]; 6(4):15-34. Disponível em: http://revista.cofen.gov.br/index.php/enfermagem/article/viewFile/687/297.

24. Rodrigues RCV, Peres HHC. Panorama brasileiro do ensino de Enfermagem On-line. Rev. Esc. Enferm. USP [internet]. 2008 [acesso em 2018 jul 16]; 42(2):298-304. Disponível em: http://www.scielo.br/scielo.php?script=sci_arttext $\&$ pid $=$ S0080$-62342008000200013 \& \operatorname{lng}=$ en. $\quad$ http://dx.doi. org/10.1590/S0080-62342008000200013.

25. World Health Organization. All-Party Parliamentary Group on Global Health (APPG). Triple Impact How developing nursing will improve health, promote gender equality and support economic growth [internet]. 2016 [acesso em 2018 jul 14]. Disponível em: http://www.appg.globalhealth.org.uk/.

26. Nursing Now. Raising the profile and status of nursing worldwide. Nursing Now [internet]. [acesso em 2018 jul 14]. Disponível em: http://www.nursingnow.org/.

27. World Health Organization. Transforming and scaling up health professionals education and training. Genova: World Health Organization 2013.
28. World Health Organization. Global strategic directions for strengthening nursing and midwifery 2016-2020 [internet]. 2016 [acesso em 2018 jul 14]. Disponível em: http://www.who.int/hrh/nursing midwifery/global-strategic-midwifery2016-2020. pdf.

29. Cassiani SH, García AB, Caballero E, et al. Redes internacionais de enfermagem nas américas: trabalho colaborativo para o alcance da cobertura universal em saúde. Enfer. Cuid. Humaniz [internet]. [acesso em 2018 jul 14]; 3. Disponível em: http://enfermeriacuidadoshumanizados.ucu.edu.uy/inicio/ item $/ 40$.

30. Secretaria Municipal de Saúde. Prefeitura de Florianópolis [internet]. [acesso em 2018 jul 14]. Disponível em: http://www.pmf.sc.gov.br/entidades/saude/index.php?cms=protocolos + de + enfermagem \& menu=10\&submenuid=1478.

31. Pires ROM. Complexidade em Saúde da Família e formação do futuro profissional de saúde. Interface - Comunic. Saude. Educ. [internet] 2009 [acesso em 2018 jul 14]; 13(30):153-66. Disponível em: http://www.scielo.br/scielo.php?script=sci arttext\&pid=S1414-32832009000300013\&lng=pt\&t lng=pt.

32. Girardi SN, Wan Der Maas L, Carvalho CL, et al. Estrutura Atual e Estimativas Futuras da Força de Trabalho em Medicina, Enfermagem e Odontologia no Brasil 2000 a 2030. Rio e Janeiro: Fundação Oswaldo Cruz; 2015.

33. ABEn Nacional [internet]. [acesso em 2018 jul 14]. Disponível em: http://www.abennacional.org.br/.

34. Royal College of Nursing. eHealth: the future of health care Royal College of Nursing position statement [internet]. London; 2016 [acesso em 2018 jul 16]. Disponível em: https://www.rcn.org.uk/ professional-development/publications/pub-004186\#copyrightTab.

35. Black AD, Car J, Pagliari C, et al. The Impact of eHealth on the Quality and Safety of Health Care: A Sys- 
tematic Overview. PLoS Med [internet]. 2011 [acesso em 2018 jul 16]; 18;8(1):e1000387. Disponível em: http://www.haps.bham.ac.uk/publichealth/cfhep/.

36. Brasil. Ministério da Educação. Enade 2016 exame nacional de desempenho dos estudantes relatório síntese da área enfermagem. 2016 [acesso 2018 jul 14]; Disponível em: http://download.inep.gov.br/ educacao_superior/enade/relatorio_sintese/2016/ enfermagem.pdf.

37. Institute of Medicine. The future of nursing : leading change, advancing health. Washington, D.C: National Academies Press; 2011.

38. McPake B, Squires A, Agya M, et al. The Economics of Health Professional Education and Careers: Insights from a Literature Review. The World Bank [internet]. 2015 [acesso em 2018 out 9]. Dis- ponível em: http://elibrary.worldbank.org/doi/

book/10.1596/978-1-4648-0616-2.

39. Frenk J, Chen L, Bhutta ZA, et al. Health professionals for a new century: transforming education to strengthen health systems in an interdependent world. Lancet [Internet]. 2010 [acesso em 2018 jun 29]; 376(9756):1923-1958. Disponível em: http:// www.ncbi.nlm.nih.gov/pubmed/21112623.

40. Crisp N, Chen L. Global Supply of Health Professionals. N Engl J Med [Internet]. 2014 [acesso em 2018 out 9]; 370(10):950-957. Disponível em: http:// www.nejm.org/doi/abs/10.1056/NEJMral111610.

Recebido em 18/07/2018

Aprovado em 25/08/2018

Conflito de interesses: inexistente

Suporte financeiro: não houve 